\title{
Conformational polymorphism in sofosbuvir: some structural insights
}

\author{
Hemant Malhari Mande ${ }^{1}$, Ram K R Jetti ${ }^{1}$, Partha $\mathrm{P}$ das $^{2}, \mathrm{~S}$. Nicolopoulos ${ }^{2}$ \\ ${ }^{1}$ Chemical Research Division, API-R\&D, Mylan Laboratories Limited, Hyderabad, India, ${ }^{2}$ NanoMEGAS SPRL, Brussels, Belgium \\ E-mail: hemant.mande@mylan.in
}

Polymorphism plays a vital role in the development of pharmaceutical ingredients. Polymorphs of active pharmaceutical ingredients (APIs) have always drawn attention in view of their physicochemical and intellectual property. Sofosbuvir (commercially marketed as Sovaldi by Gilead Sciences, USA) is one of the best-known and most prescribed drug for chronic hepatitis $C$, genotypes $1,2,3,4,5$, and 6 , usually in combination with other medications depending on the specific genotype. Sofosbuvir is a prodrug that is metabolized to the active antiviral agent 2 '-deoxy-2'-a-fluoro- $\beta$-C-methyluridine$5^{\prime}$-monophosphate. It is a high revenue API ( $\$ 24.9$ billion in 2014) since was first commercialized in 2013. Sofosbuvir is known to exhibit polymorphism[1] and till date more than 15 polymorphs including amorphous form are reported. Gilead Sciences reported[1a-1c] eight crystalline polymorphs, 4 anhydrous forms (designated as Form 1, Form 6, Form 7 and Form 8 ) and 4 unstable solvates of Sofosbuvir. The four anhydrous forms showed varying melting points in the range of 100 to $140^{\circ} \mathrm{C}$. Recently Qi et al [1k] reported thermodynamic relationships of Sofosbuvir polymorphs by thermal analysis and solubility extrapolation and confirms that form 6 and Form 7 are enantiotropic in nature while form 1 is monotropic in nature with form 6 and form 7. However, till date no report on crystal structures of Sofosbuvir polymorphs were known in literature. We report here for the first time crystal structure analysis of Sofosbuvir polymorphs Form 1 , Form 7 and Form 8. Form 1 and form 7 are crystallizes in monoclinic crystal (P21) system while form 8 get crystallized into orthorhombic crystal system with non-centrosymmetric space group P212121. The crystal structure analysis reveals Sofosbuvir exhibits conformational polymorphism. Conformational flexibility around phosphate group leads to different types of hydrogen bonding networks dominated by $\mathrm{N}-\mathrm{H} . . . \mathrm{O}, \mathrm{O}-\mathrm{H} \ldots \mathrm{O}, \mathrm{C}-\mathrm{H} \ldots \mathrm{O}$ interactions. Further, the inability to grow the single crystals of Form 6 suitable for single crystal X-ray diffraction made us to explore 3D precession electron diffraction tomography[2] and rietveld method[3] for deriving the crystal structure. In this work we discuss for the first time, structural features of Sofosbuvir polymorphs and application of electron diffraction technique for crystal structure determination of Form 6.

[1] International patent publication number (a) US9206217B2 (b) US8618076B2 (c) US20150175646A1 (d) W02015126995A1 (e) WO2015/191945A2 (f) CN104447924A1 $\quad$ (g) CN104804054A1 (h) CN104650171A1 (i) CN104277088A1 (j) W02016038542A2 (k) Ming-Hui Qi, Ming-Huang Hong, Yan Liu, En-Fu Wang, Fu-Zheng Ren, \& Guo-Bin Ren (2015) Cryst. Growth Des, 15 (10), 5062-5067.

[2] (a) Van Genderen, E., Clabbers, M. T. B., Das, P. P., Stewart, A., Nederlof, I., Barentsen, K. C., Portillo, Q., Pannu, N. S., Nicolopoulos, S., Gruene, T. \& Abrahams, J. P. ( 2016) Acta Cryst. A72, 236-242; (b) Kolb U., Gorelik T., Keble C., Otten M. T \& Hubert D., (2007) Ultramicroscopy 107, 507-513.

[3] TOPAS V5: General Profile and Structure Analysis Software for Powder Diffraction Data; Bruker AXS GmbH: Karlsruhe, Germany, 2014.

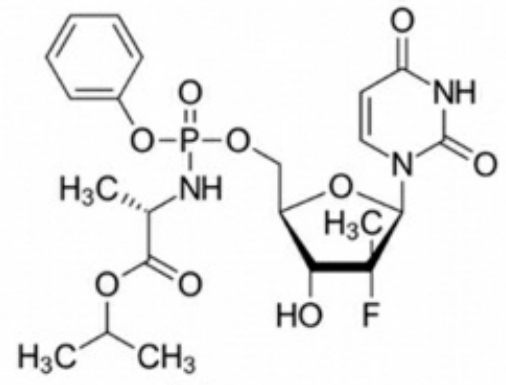

(a)

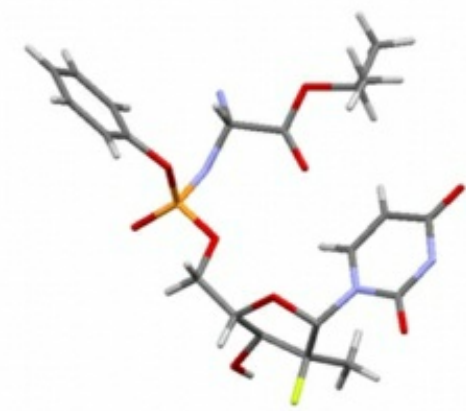

(b)

Fig. 1: (a) Structure of Sofosbuvir; (b) Single crystal X-ray structure of Sofosbuvir

Keywords: Polymorphism, Single crystal X-ray Diffraction, 3D-Precession electron diffraction tomography 